\title{
Clobetasol down-regulates SLPI expression in U937 monocytoid cells
}

\author{
NAOKO OKUMURA*, HITOMI YOSHIDA*, YASUKO KITAGISHI, YURI NISHIMURA and SATORU MATSUDA \\ Department of Environmental Health Science, Nara Women's University, Nara 630-8506, Japan
}

Received September 5, 2011; Accepted October 24, 2011

DOI: $10.3892 / \mathrm{ijmm} .2011 .825$

\begin{abstract}
In order to investigate how glucocorticoids affect the expression of secretory leukocyte peptidase inhibitor (SLPI), which is overexpressed in a variety of cancers, clobetasol was added to cell culture medium of U937 cells and the SLPI mRNA levels were examined. The in vitro effect of the treatment on SLPI expression was detected by reverse transcriptase-polymerase chain reaction. Clobetasol treatment of U937 cells induced an up- and down-regulation of SLPI expression in a dose-dependent manner. Western blotting confirmed the down-regulation of SLPI protein expression. We hypothesized a loop formation in the SLPI genome domain, in which the glucocorticoid receptor regulates bi-directional transcriptional activity.
\end{abstract}

\section{Introduction}

Secretory leukocyte peptidase inhibitor (SLPI) is a $12 \mathrm{kDa}$ serine protease inhibitor, which is minimally produced by normal epithelial cells lining the respiratory, digestive, and reproductive tracts $(1,2)$. SLPI is overexpressed in a variety of cancers, but its role in cancer is not well understood. SLPI genes are often up-regulated under tumorigenic conditions. Overexpression of this protein correlates with more-invasive forms of the cancer (3). The expression levels of SLPI gene have been associated to both disease-free and overall survival (4). In addition, gene-targeting experiments in mice have indicated that carcinogenesis is suppressed in the SLPI knockout mice (5). Accordingly, repression of SLPI expression may be expected to have interference effects on cancer progression. The SLPI gene is regulated by glucocorticoids, which play an essential role in embryonic and cancer development (6). Due to the wide spectrum of activity and pro-apoptotic

Correspondence to: Dr Satoru Matsuda, Department of Environmental Health Science, Nara Women's University Kita-Uoya Nishimachi, Nara 630-8506, Japan

E-mail: smatsuda@cc.nara-wu.ac.jp

${ }^{*}$ Contributed equally

Key words: secretory leukocyte peptidase inhibitor, clobetasol, glucocorticoid receptor, gene expression, cancer properties, glucocorticoids are one of the most commonly drugs used in hematological malignancies and are also used as co-treatment chemotherapy regimens in other types of cancers. Glucocorticoid receptor (GR) is a member of the nuclear hormone receptor family (7). Hormone activated GR binds to glucocorticoid responsive elements (GRE) near the target genes (8). However, glucocorticoids could play a complex role in cancer epidemiology, biology and treatment (9).

\section{Materials and methods}

Cell culture. The human cell lines U937 and K562 were maintained in RPMI-1640 supplemented with $10 \%$ fetal bovine serum (FBS), penicillin and streptomycin at $37^{\circ} \mathrm{C}$ in a humidified atmosphere containing $5 \% \mathrm{CO}_{2}$.

Reagents preparation. Reagents used were dissolved in ethanol and subsequently diluted at a stock concentration of $10 \mathrm{mM}$. It was stored at $-20^{\circ} \mathrm{C}$ until use. For the cell treatments, a range of 0.5-10.0 $\mu \mathrm{l}$ was added into $1 \mathrm{ml}$ of cell culture medium.

Reverse transcriptase-polymerase chain reaction (RT-PCR). SLPI and GAPDH mRNAs were analyzed by semi-quantitative RT-PCR. Total-RNA was extracted by an RNA isolation kit (Takara, Japan). Total-RNA ( $2 \mu \mathrm{g})$ was reverse-transcribed using a Phusion RT-PCR kit (NEB) as described in the manufacturer's protocol. Cycle-based PCR was used to semiquantitate the ubiquilin1 and presenilin1 gene level. GADPH was also used as an internal loading control. All the samples were determined within 3 months after collection. Thermocycling was performed according to the manufacturer's instructions at $55^{\circ} \mathrm{C}$ annealing temperature in a final volume of $10 \mu \mathrm{l}$ including TaqDNA polymerase.

Western blot analysis. Equal amounts of protein samples were used for Western blot analysis using anti-SLPI (Abnova) and anti-Erk2 (AnaSpec, Inc.) antibodies, and quantified by densitometry. All the Western blot analyses were repeated at least 3 times and the representative data are shown.

\section{Results and Discussion}

In order to investigate how glucocorticoids affect the expression of SLPI, clobetasol as well as dexamethasone were added into the cell culture medium of U937 or K562 


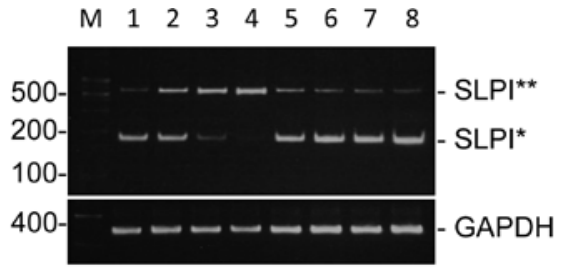

(bp)

Figure 1. Semi-quantitative RT-PCR was performed using primers specific to SLPI on $100 \mathrm{ng}$ total-RNA prepared from U937 cells treated without (lane 1, lane 5) or with clobetasol propionate (Sigma) at the final concentration $10^{-7} \mathrm{M}$ (lane 2), $10^{-6} \mathrm{M}$ (lane 3), $10^{-5} \mathrm{M}$ (lane 4) or with dexamethasone (Sigma) at the final concentration $10^{-7} \mathrm{M}$ (lane 6), $10^{-6} \mathrm{M}$ (lane 7), $10^{-5} \mathrm{M}$ (lane 8) for $24 \mathrm{~h}$. Specific expression was determined in relation to the expression of the housekeeping gene GAPDH used as an internal loading control. At least 4 independent experiments were done, and a typical paired result is documented.

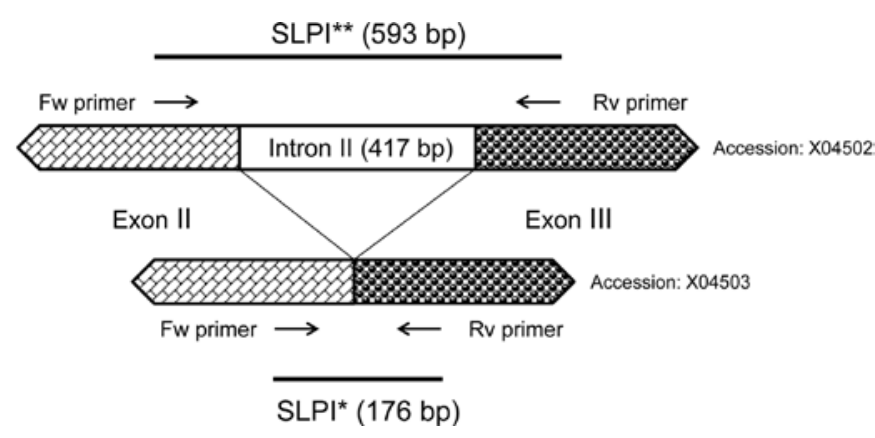

Figure 2. Schematic representation of the SLPI genome structure between exon 2 and exon 3, and the site for primers used in the RT-PCR experiment. The predicted PCR products (SLPI ${ }^{*}$ and SLPI ${ }^{* *}$ correspond to short and long bands in Fig. 1, respectively) by sequencing are depicted.

cells and the levels of SLPI expression were examined. We employed RT-PCR analysis to quantify the expression level of the SLPI gene. Total-RNA was extracted $24 \mathrm{~h}$ after treatment for detection of the SLPI, and was reverse-transcribed. The levels of mRNA were determined by the conventional RT-PCR using primers specific to SLPI forward, 5'-CTGCCCAGTGCCTTAGATACA-3' and reverse, 5'-CATC AAACATTGGCCATAAGT-3', (expected size, $176 \mathrm{bp}$ ); and GAPDH forward, 5'-TCCCATCACCATCTTCCA-3' and reverse, 5'-CATCACGCCACAGTTTCC-3', (expected size, $376 \mathrm{bp})$.

As shown in Fig. 1, there were 2 sizes of band for SLPI expression by the RT-PCR. After pre-treating the cells with a set of different concentrations of clobetasol, we found that expression levels of the short band (SLPI $\left.{ }^{*}\right)$ greatly decreased, whereas those of the long band (SLPI $\left.{ }^{* *}\right)$ greatly increased, in a dose-dependent manner in U937 monocytoid cells. However, gene expression of the two SLPI bands was unaltered with increasing concentrations of dexamethasone compared with the untreated ethanol vehicle. A larger amount of dexamethasone $\left(10^{-4} \mathrm{M}\right)$ could reduce the expression levels of the SLPI band and could increase SLPI ${ }^{* *}$ gene expression (data not shown), probably because of the efficacy difference of glucocorticoids. On the contrary, the expression of the housekeeping gene GAPDH was also unaltered. There was almost no difference in the results of gene expression between U937 and K562



Figure 3. Dose-dependent repression of SLPI protein expression by clobetasol U937 cells were treated without (lane 1) or with clobetasol propionate at a final concentration of $10^{-8} \mathrm{M}$ (lane 2), $10^{-7} \mathrm{M}$ (lane 3), $10^{-6} \mathrm{M}$ (lane 4), $10^{-5} \mathrm{M}$ (lane 5), $10^{-4} \mathrm{M}$ (lane 6) for $48 \mathrm{~h}$. The levels of SLPI protein were detected by Western blot analysis using anti-SLPI. Western blotting with anti-Erk2 antibody was also shown as equal levels of protein loading.



Figure 4. Schematic representation of a hypothetical loop formation in the SLPI domain. The glucocorticoid receptor regulates bi-directional transcriptional activity of both promoters by mechanisms related to the loop.

cells. To exclude the possibility of carry-over contamination, reactions containing all RT-PCR reagents including primers without sample RNA were performed as negative controls. No such contamination was detected (data not shown). Sequencing of the excised bands from the analysis gel revealed that the short SLPI band was the SLPI exon as expected, and that the long one included intron II in addition to the short one. The structures of the 2 bands are illustrated in Fig. 2. There was no band found with RT-PCR using the primers within either intron I or intron III of the SLPI genome even with similar clobetasol stimulation (data not shown).

To further confirm the expression status of SLPI, Western blotting methods with anti-SLPI and anti-Erk2 antibodies were performed to analyze the level of SLPI protein in U937 cells. As shown in Fig. 3, after pre-treating the cells with a set of different doses of clobetasol, we found that the SLPI protein expression was decreased with increasing concentrations of clobetasol. A final concentration $10^{-5} \mathrm{M}$ of the clobetasol diminished the SLPI protein expression by more than $90 \%$ after $48 \mathrm{~h}$ stimulation. This protein expression profile approximately agreed with the result of the short band of RT-PCR shown in Fig. 1.

The kinetics of the GR-stimulated transcriptional output is very complex, and different cell types vary in their response to GR $(10,11)$. It has been suggested that loop formation of GR responsive genome structure plays a role in transcriptional regulation (12). In the loop structure, specific long range interactions may play a role in GR function (12). It is plausible that the long size of RT-PCR product in Fig. 1 might be transcribed reversely from a site downstream the SLPI gene in the genomic loop (Fig. 4). Examinations of multiple sets of 
GR-regulated genes will be required to explore the validity of the concept. There is accumulating evidence that a lack of SLPI in cancer cells suppresses tumor formation, and that SLPI is up-regulated in tumor formation $(13,14)$. Here, we show that clobetasol is a potent transcriptional repressor for SLPI expression. The precise mechanism of transcriptional regulation of SLPI by clobetasol remains unclear. SLPI may be regulated by various factors. More studies including in vivo experiments need to be undertaken to elucidate the molecular mechanisms, and further analysis of SLPI function in relation to the tumor forming capacity may lead to the development of a novel treatment modality.

\section{Acknowledgements}

This study was supported by grants-in-aid from the Ministry of Education, Culture, Sports, Science and Technology in Japan and a Nara Women's University Intramural grant for Project Research. In addition, this study was supported in part by a grant from the Fuji Foundation for Protein Research.

\section{References}

1. Fath MA, Wu X, Hileman RE, Linhardt RJ, Kashem MA, Nelson RM, Wright CD and Abraham WM: Interaction of secretory leukocyte protease inhibitor with heparin inhibits proteases involved in asthma. J Biol Chem 273: 13563-13569, 1998.

2. Wahl SM, McNeely TB, Janoff EN, Shugars D, Worley P, Tucker C and Orenstein JM: Secretory leukocyte protease inhibitor (SLPI) in mucosal fluids inhibits HIV-I. Oral Dis 3 (Suppl 1): S64-S69, 1997.

3. Merritt MA, Parsons PG, Newton TR, Martyn AC, Webb PM, Green AC, Papadimos DJ and Boyle GM: Expression profiling identifies genes involved in neoplastic transformation of serous ovarian cancer. BMC Cancer 9: 378, 2009.
4. Cimino D, Fuso L, Sfiligoi C, Biglia N, Ponzone R, Maggiorotto F, Russo G, Cicatiello L, Weisz A, Taverna D, Sismondi P and De Bortoli M: Identification of new genes associated with breast cancer progression by gene expression analysis of predefined sets of neoplastic tissues. Int J Cancer 123: 1327-1338, 2008.

5. Nukiwa T, Suzuki T, Fukuhara T and Kikuchi T: Secretory leukocyte peptidase inhibitor and lung cancer. Cancer Sci 99: 849-855, 2008

6. Hayashi R, Wada H, Ito K and Adcock IM: Effects of glucocorticoids on gene transcription. Eur J Pharmacol 500: 51-62, 2004

7. Gross KL and Cidlowski JA: Tissue-specific glucocorticoid action: a family affair. Trends Endocrinol Metab 19: 331-339, 2008.

8. Kumar R and Thompson EB: Gene regulation by the glucocorticoid receptor: structure:function relationship. J Steroid Biochem Mol Biol 94: 383-394, 2005.

9. Vaidya JS, Baldassarre G, Thorat MA and Massarut S: Role of glucocorticoids in breast cancer. Curr Pharm Des 16: 3593-3600, 2010.

10. John S, Johnson TA, Sung MH, Biddie SC, Trump S, Koch-Paiz CA, Davis SR, Walker R, Meltzer PS and Hager GL: Kinetic complexity of the global response to glucocorticoid receptor action. Endocrinology 150: 1766-1774, 2009.

11. Biddie SC, John S and Hager GL: Genome-wide mechanisms of nuclear receptor action. Trends Endocrinol Metab 21: 3-9, 2010.

12. Hakim O, John S, Ling JQ, Biddie SC, Hoffman AR and Hager GL: Glucocorticoid receptor activation of the Ciz1-Len2 locus by long range interactions. J Biol Chem 284: 6048-6052, 2009.

13. Wen J, Nikitakis NG, Chaisuparat R, Greenwell-Wild T, Gliozzi M, Jin W, Adli A, Moutsopoulos N, Wu T, Warburton G and Wahl SM: Secretory leukocyte protease inhibitor (SLPI) expression and tumor invasion in oral squamous cell carcinoma. Am J Pathol 178: 2866-2878, 2011.

14. Cheng WL, Wang CS, Huang YH, Liang Y, Lin PY, Hsueh C, Wu YC, Chen WJ, Yu CJ, Lin SR and Lin KH: Overexpression of a secretory leukocyte protease inhibitor in human gastric cancer. Int J Cancer 123: 1787-1796, 2008. 